\title{
Nodular pulmonary amyloidosis with unusual, widespread lung cysts
}

Kian Ming ${\underline{C h e w^{1}}}^{1}$ MBChB, FRCR, Michael John $\underline{\text { Clarke }}^{1}$, MBBS, FRANZCR, Niraj Dubey ${ }^{1}$, MBBS, FRCR, Ju Ee Seet $^{2}$, MBChB, FRCPath

\begin{abstract}
A 49-year-old Chinese woman was referred to our hospital for management of multiple lung nodules, which were incidentally detected on routine chest radiography. Chest computed tomography (CT) confirmed the presence of multiple pulmonary nodules and lung cysts. The lung cysts were located apart from the pulmonary nodules. CT-guided biopsy of the pulmonary nodule specimens confirmed the diagnosis of nodular pulmonary amyloidosis. While cavitation of existing pulmonary amyloid nodules is a well-recognised feature of nodular pulmonary amyloidosis, widespread lung cysts located apart from pulmonary nodules is rare.
\end{abstract}

Keywords: amyloidosis, computed tomography, pulmonary cysts, pulmonary nodules

\section{INTRODUCTION}

As a heterogenous group of disorders, amyloidosis is associated with the deposition of abnormal protein formed by amyloid fibril in the extracellular tissue. Localised amyloidosis refers to amyloid deposition in a single organ, such as the lung, while concurrent involvement with and deposition in other organs is described as systemic amyloidosis. Nodular parenchymal pulmonary amyloidosis is thus classified as a form of localised pulmonary amyloidosis. Pulmonary nodules, with or without calcification and cavitation, are common imaging findings. We describe an unusual case of nodular pulmonary amyloidosis where multiple lung cysts were a dominant feature besides lung nodules.

\section{CASE REPORT}

A 49-year-old Chinese woman with no significant past medical history was referred to a respiratory clinic for further investigation of abnormal chest radiography findings detected on routine health screening. The patient was completely asymptomatic and denied having any cough, fever, haemoptysis, dyspnoea, reduced effort tolerance or recent loss of weight. No smoking history or family history of lung cancer was elicited. On physical examination, the patient appeared well nourished. There were no signs of anaemia or clubbing. Auscultation of the lungs revealed good air entry bilaterally with no rhonchi. There were no palpable cervical, supraclavicular or axillary lymph nodes. Examination of the breast and abdomen was also unremarkable.

Repeat chest radiography in our institution revealed the presence of multiple, irregular nodular opacities in both lungs, which were associated with scattered areas of cystic change with no definite zonal predilection (Fig. 1). There was no increase in interstitial markings or any significant loss of lung volume. Further evaluation with intravenous contrast-enhanced computed tomography (CT) of the thorax, abdomen and pelvis confirmed

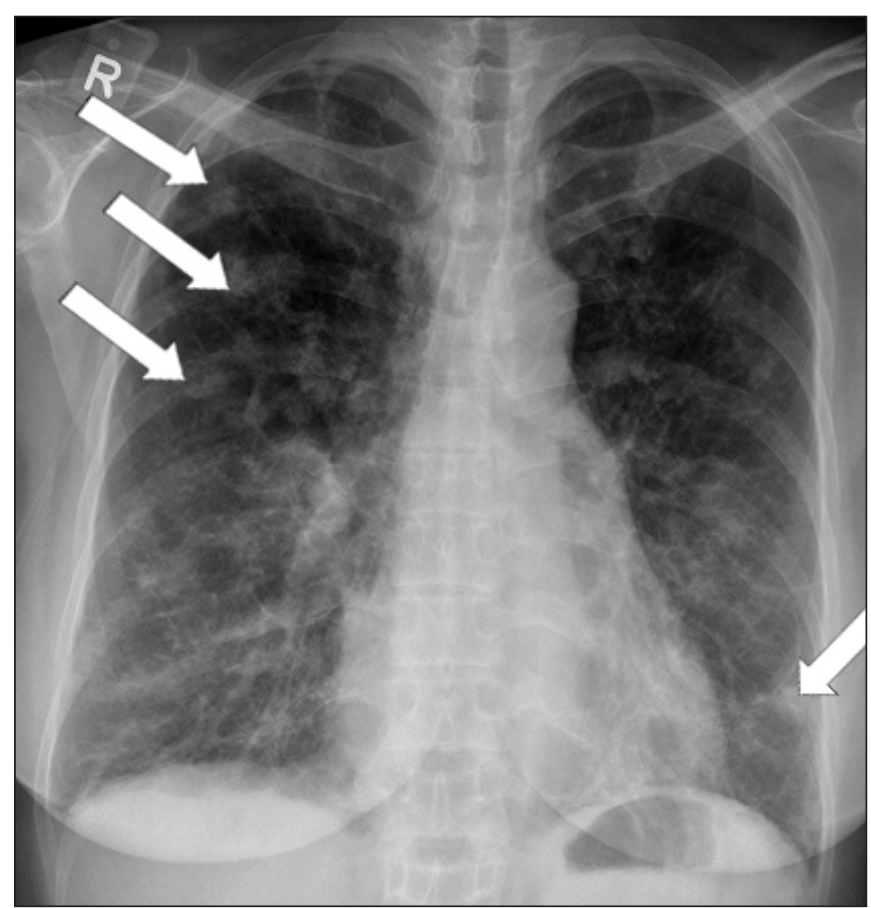

Fig. 1 Chest radiograph shows multiple, bilateral, irregular nodular opacities (arrows), which are more abundant on the right.

the presence of multiple nodules in both lungs, with measurements ranging from 2-15 mm. Multiple bilateral lung cysts of various sizes in the central and subpleural regions were also present (Fig. 2). There was no pleural effusion or pneumothorax. No enlarged mediastinal lymph nodes were demonstrated and no abnormality in the abdomen was seen. The patient subsequently underwent CT-guided core biopsy of her right upper lobe subpleural nodule. The core biopsies (one is shown in Fig. 3) showed dense aggregates of acellular, homogeneous, eosinophilic material. The material was periodic acid-Schiff positive. Congo red staining showed salmon pink staining on light microscopy and apple-green birefringence on polarised

${ }^{1}$ Department of Diagnostic Radiology, Khoo Teck Puat Hospital, ${ }^{2}$ Department of Pathology, National University Hospital, Singapore

Correspondence: Dr Chew Kian Ming, Registrar, Department of Diagnostic Radiology, Khoo Teck Puat Hospital, 100 Yishun Central, Singapore 768826. ckianming@hotmail.com 

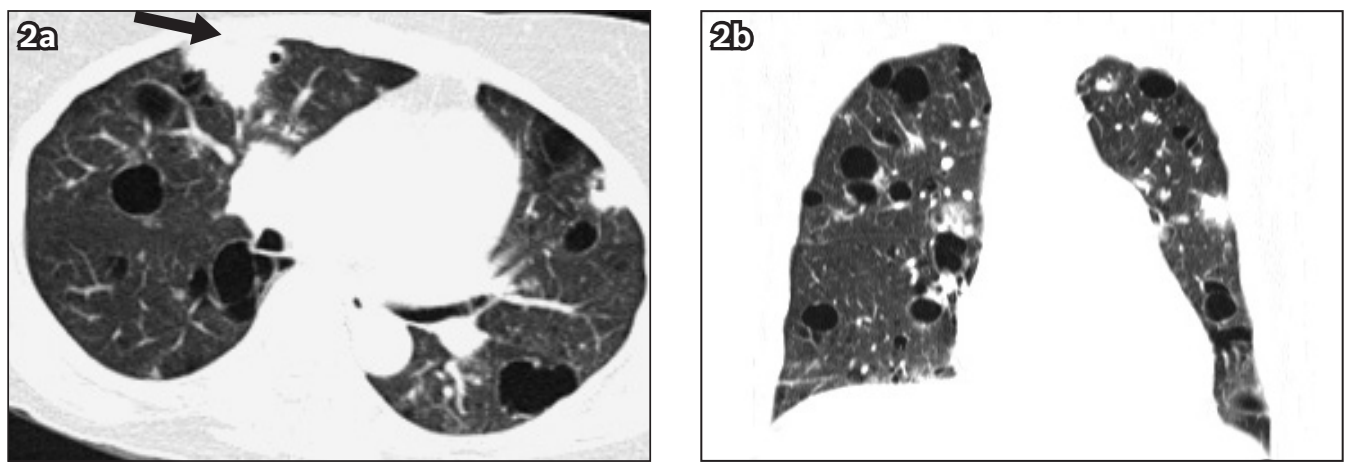

Fig. 2 (a) Axial and (b) coronal chest CT images show multiple, bilateral nodularities and cavities with no zonal predilection. The dominant nodule in the subpleural region of the right upper lobe (arrow) was biopsied.
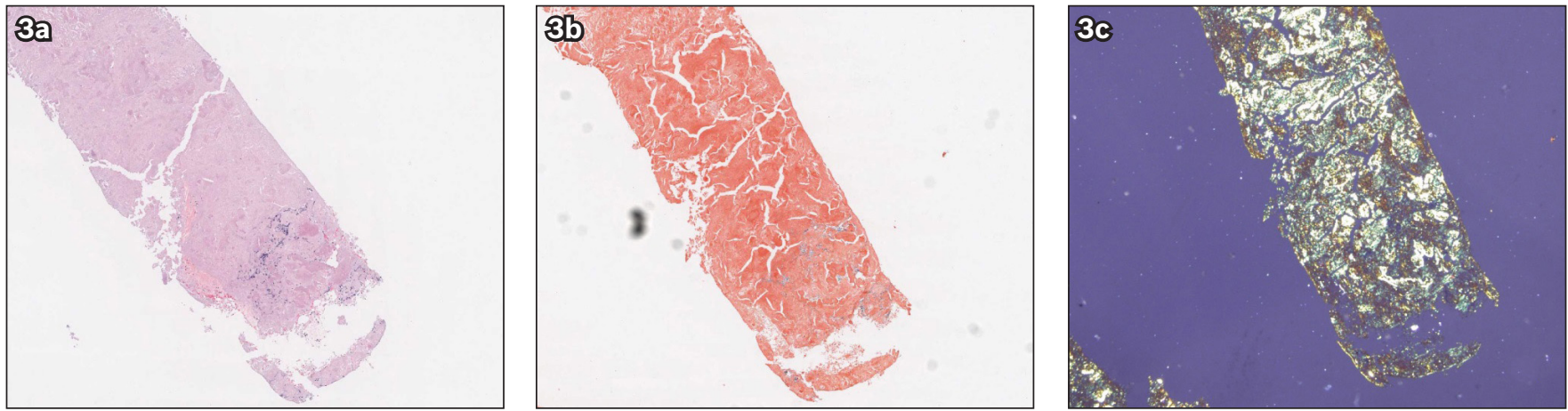

Fig. 3 Photomicrographs under (a \& b) light microscopy and (c) polarised light microscopy show core biopsy of the lung ([a] Haematoxylin \& eosin, $\times 40 ;$ [b] Congo red, $\times 40$; [c] Congo red, $\times 40$ ).

light microscopy. There was also positive immunohistochemical staining for amyloid protein $\mathrm{P}$ and positive immunofluorescence with thioflavin T. Overall, histological findings were consistent with localised nodular pulmonary amyloidosis.

\section{DISCUSSION}

Amyloidosis is a heterogenous group of disorders associated with extracellular deposition of protein in various organs in an abnormal fibrillar form. Characteristically, amyloid deposits show apple-green birefringence when stained with Congo red and viewed under polarised light. Amyloidosis may present in systemic or localised forms, and pulmonary involvement may be localised, as in localised pulmonary amyloidosis, or represent part of a systemic process (systemic amyloidosis). Systemic amyloidosis can be further classified into primary and secondary systemic amyloidosis. Primary systemic amyloidosis is associated with an underlying monoclonal dyscrasia of plasma or B-lymphoid cells. Deposition of amyloid can occur in various organ systems, with the gastrointestinal system, heart and kidneys being common sites of involvement. However, secondary systemic amyloidosis is associated with chronic conditions such as tuberculosis, chronic renal disease, rheumatoid arthritis and ankylosing spondylitis. Pulmonary involvement in systemic amyloidosis is not uncommon, as demonstrated in studies by Utz et al ${ }^{(1)}$ and Smith et al. ${ }^{(2)}$ Utz et al's ${ }^{(1)}$ study reported poor prognosis in 21 patients with primary systemic amyloidosis, with a median survival of 16 months.

Localised pulmonary amyloidosis is defined as deposition of amyloid isolated to the respiratory tract, with no evidence of systemic disease. According to the location and pattern of involvement, three main forms of localised pulmonary amyloidosis have been described in the literature: (a) tracheobronchial amyloidosis; (b) diffuse interstitial amyloidosis; and (c) nodular pulmonary amyloidosis. Tracheobronchial amyloidosis is characterised by the presence of tumour-like nodules, multiple, discrete plaques, or circumferential thickening (with or without luminal narrowing of the trachea, main bronchi, and lobar or segmental bronchi). These nodules or plaques may be localised or multifocal. ${ }^{(3,4)}$ Depending on the size and location of the nodules, patients may be asymptomatic or present with symptoms of airway obstruction such as wheeze, dyspnoea, cough, haemoptysis and recurrent chest infection. Death is usually secondary to progressive bronchial obstruction and subsequent respiratory failure. Mural nodules and calcification of the thickened tracheobronchial wall are common CT findings. ${ }^{(4,5)}$ Diffuse interstitial amyloidosis is the least common of the three main forms of localised pulmonary amyloidosis and has the worst prognosis, with widespread deposition of amyloid involving small vessels and parenchymal interstitium. While Hui et al ${ }^{(6)}$ noted a diffuse interstitial pattern in six of 48 localised pulmonary amyloidosis cases, no such case was reported by Utz et $\mathrm{al}^{(1)}$ in their cohort consisting of 17 patients with localised pulmonary amyloidosis. Radiographic findings include ground-glass infiltrates, abnormal reticular opacities, interlobular septal thickening, nodules of 2-4 $\mathrm{mm}$ in size and consolidation in the subpleural region. ${ }^{(4,5)}$ Nodular pulmonary amyloidosis (seen in the present case) carries the most favourable prognosis and long-term survival is expected even without treatment. Patients 
are typically asymptomatic and incidental findings of pulmonary nodules on routine chest radiographs are a common mode of presentation, although some may present with cough, shortness of breath and haemoptysis. However, a solitary nodule is a more common finding than multiple nodules on chest radiograph. On CT, pulmonary nodules have sharp and lobulated margins, and are usually found in a subpleural or peripheral location, more frequently in the lower lobes. The size of the nodules may range from micronodular to $15 \mathrm{~cm}$ in diameter. ${ }^{(3,4)}$ Over time, nodules may grow slowly and cavitate, calcify, or resolve spontaneously. ${ }^{(3,7,8)}$ Calcification can be seen in $20 \%-50 \%$ of nodules on CT images. ${ }^{(4)}$

In our patient, the appearance and location of the nodules are similar to those described by various authors. ${ }^{(1,4,5)}$ What is unusual in our patient is the widespread cyst formation in her lungs, which was associated with nodular pulmonary amyloidosis (Fig. 2b). The cysts were thin-walled and located apart from the lung nodules, and there was no cavitation of the visualised pulmonary nodules to suggest that the cysts were secondary to extensive cavitation of nodules. Lantuejoul et al( ${ }^{(9)}$ reported a case of nodular pulmonary amyloidosis with similar cystic change in the lungs, and association with mucosa-associated lymphoid tissue lymphoma was confirmed on surgical lung biopsy. Sjögren's syndrome complicated by multiple bullae and nodular pulmonary amyloidosis has also been described. ${ }^{(10-12)}$ A case of primary diffuse alveolar septal amyloidosis with multiple cyst formation was reported by Ohdama et al. . $^{(13)}$ In contrast to reported cases, core biopsy specimens obtained in our case showed no evidence of lymphoid proliferation. Our patient also did not have clinical signs or symptoms of Sjögren's syndrome. Ishibashi et al ${ }^{(14)}$ had also reported a case of nodular pulmonary amyloidosis associated with bullae, but without any underlying inflammatory or proliferative disorder. However, the disease in Ishibashi et al's ${ }^{(14)}$ case was localised in the right lower lung zone, as opposed to diffuse bilateral involvement seen in our patient.

The mechanism of cyst formation in the presence of pulmonary amyloidosis is unclear. However, three possible mechanisms leading to cyst formation have been postulated: (a) narrowing of the airway with inflammatory cell infiltration, resulting in check valve mechanism; (11) (b) disruption of alveolar walls secondary to deposition of amyloid within broncholoalveolar structures; ${ }^{(13)}$ and (c) deposition of amyloid around capillaries and within interstitial tissue, possibly resulting in obliteration of alveolar capillaries, causing ischaemia and destruction of alveolar walls. ${ }^{(15)}$ Also, due to the nonspecific appearance of pulmonary amyloidosis, diagnosis requires histological confirmation. Congo red staining produces characteristic apple-green birefringence under polarised light in the presence of amyloidosis.

In conclusion, pulmonary amyloidosis is a rare disease entity that should always be considered in patients presenting with multiple lung nodules. The condition's association with widespread lung cysts, as described in our case, is an unusual finding, particularly in the absence of associated inflammatory or proliferative disorders. Although localised pulmonary amyloidosis carries a more favourable prognosis than its systemic counterpart, regular follow-up is recommended to monitor the progress of the pulmonary lesions and development of any respiratory symptoms, in order to initiate early treatment when necessary.

\section{REFERENCES}

1. Utz JP, Swensen SJ, Gertz MA. Pulmonary amyloidosis. The Mayo Clinic experience from 1980 to 1993. Ann Intern Med 1996; 124:407-13.

2. Smith RR, Hutchins GM, Moore GW, Humphrey RL. Type and distribution of pulmonary parenchymal and vascular amyloid. Correlation with cardiac amyloid. Am J Med 1979; 66:96-104.

3. Gillmore JD, Hawkins PN. Amyloidosis and the respiratory tract. Thorax 1999; 54:444-51.

4. Chung MJ, Lee KS, Franquet T, et al. Metabolic lung disease: imaging and histopathologic findings. Eur J Radiol 2005; 54:233-45.

5. Georgiades CS, Neyman EG, Barish MA, Fishman EK. Amyloidosis: review and CT manifestations. Radiographics 2004; 24:405-16.

6. Hui AN, Koss MN, Hochholzer L, Wehunt WD. Amyloidosis presenting in the lower respiratory tract. Clinicopathologic, radiologic, immunohistochemical, and histochemical studies on 48 cases. Arch Pathol Lab Med 1986; 110:212-8.

7. Suzuki H, Matsui K, Hirashima T, et al. Three cases of the nodular pulmonary amyloidosis with a longterm observation. Intern Med 2006; 45:283-6.

8. Fukatsu H, Miyoshi $\mathrm{H}$, Ishiki K. Spontaneous resolution of multiple nodular pulmonary AA amyloidosis. Intern Med 2010; 49:2303-7.

9. Lantuejoul S, Moulai N, Quetant S, et al. Unusual cystic presentation of pulmonary nodular amyloidosis associated with MALT-type lymphoma. Eur Respir J 2007; 30:589-92.

10. Jeong YJ, Lee KS, Chung MP, et al. Amyloidosis and lymphoproliferative disease in Sjögren syndrome: thin-section computed tomography findings and histopathologic comparisons. J Comput Assist Tomogr 2004; 28:776-81

11. Kobayashi H, Matsuoka R, Kitamura S, Tsunoda N, Saito K. Sjögren's syndrome with multiple bullae and pulmonary nodular amyloidosis. Chest 1988; 94:438-40.

12. Miyagawa T, Mochizuki Y, Nakahara Y, et al. [A case of Sjögren syndrome with pulmonary nodular amyloidosis and pulmonary multiple cysts.] Nihon Kokyuki Gakkai Zasshi 2009; 47:737-41. Japanese.

13. Ohdama S, Akagawa S, Matsubara O, Yoshizawa Y. Primary diffuse alveolar septal amyloidosis with multiple cysts and calcification. Eur Respir J 1996; 9:1569-71.

14. Ishibashi $H$, Akamatsu $H$, Sunamori M, Ishibashi T, Iwata T. [Nodular pulmonary amyloidosis with bullae; report of a case.] Kyobu Geka 2002; 55:1069-72. Japanese.

15. Wilson SR, Sanders DE, Delarue NC. Intrathoracic manifestations of amyloid disease. Radiology 1976; 120:283-9. 